\title{
An automated nanoliter dispenser for staining individual biopsies in tissue microarrays
}

\author{
JARED SLOBODAN $^{1}$, DYLAN GUNN ${ }^{1}$, JASON THOMPSON $^{1}$, KURTIS GUGGENHEIMER ${ }^{2}$, KEDDIE BROWN $^{3}$, \\ MARK HOMENUKE ${ }^{1}$, ROY BELAK ${ }^{1}$, DIPONKAR BANERJEE ${ }^{4}$, DAVID BROEMELING ${ }^{1}$ and ANDRE MARZIALI $^{1}$ \\ ${ }^{1}$ Applied Biophysics Laboratory, The University of British Columbia, 325-6224 Agricultural Road, Vancouver, BC, V6T 1Z1; \\ ${ }^{2}$ Genome Sciences Centre, BC Cancer Agency, 675 West 10th Avenue, Vancouver, BC, V5Z 1L3; \\ ${ }^{3}$ James Hogg iCapture Centre, St. Paul's Hospital, 1081 Burrard Street, Vancouver, BC, V6Z 1Y6; \\ ${ }^{4}$ BC Cancer Agency, 675 West 10th Avenue, Vancouver, BC, V5Z 1L3, Canada
}

Received September 20, 2006; Accepted November 28, 2006

\begin{abstract}
In the field of molecular analysis of cancer, there exists a need for a clinical device that can automate protocols for immunohistochemical and in situ hybridization diagnostic staining on tissue microarrays. The Tissue Microarray Antibody Spotter (TMAS) has been developed to provide fundamental improvements over current histological staining techniques by enabling precision application of reagents to individual biopsies within a tissue microarray. This allows for multiplexed reactions on a single slide and promises to significantly reduce costs associated with immunohistochemistry and in situ hybridization based assays. Additionally, because TMAS allows for testing of different biomarkers on each element of a tissue array, a complete cancer profile can be obtained from a single TMA slide. Ultimately this may lead to costeffective, faster and more accurate diagnosis of the patient.
\end{abstract}

\section{Introduction}

Until recently, IHC and ISH analysis has been performed on relatively large tissue samples with one test performed per slide (Fig. 1). The development of tissue microarrays (TMA) has helped to parallelize IHC and ISH analysis (1-4) by allowing a single test to be performed on potentially hundreds of smaller tissue samples mounted on the same slide (Fig. 2), simply by coating the entire slide with a single biomarker. This format is useful when a large number of biopsies need to be tested for the same biomarker. However, it did not allow for testing of a large number of biomarkers, as there was no way to treat biopsies within a TMA on an individual basis.

The tissue microarray antibody spotter (TMAS) instrument maximizes the utility of the existing TMA

Correspondence to: Dr Andre Marziali, Department of Physics and Astronomy, The University of British Columbia, Room 415, 6224 Agricultural Road, Vancouver, BC, V6T 1Z1, Canada

E-mail: andre@physics.ubc.ca

Key words: immunohistochemistry, tissue microarrays, antibodies, fluid transfer, automation, cancer platform by providing more precise and versatile fluid delivery. Specifically, TMAS has been developed to allow for delivery of a different reagent to each biopsy in a TMA. This enables one to perform potentially hundreds of different assays on a single slide, and reduces the amount of antibody solution used per assay by more than a factor of 10. By allowing reagent multiplexing on a single TMA slide, TMAS can help further accelerate studies which seek to link specific biomarkers to clinical endpoints (5-7), thus increasing the number of useful diagnostic and prognostic markers that can be used in a clinical setting.

The ultimate extension of this technology will be to cytology arrays derived from a single patient's fine needle aspiration biopsy, which would allow the screening of dozens of clinically relevant biomarkers simultaneously on a single slide in an array-based multiplexed IHC/FISH protocol. This will enable multi-biomarker profiling of specimens with limited cell numbers in routine clinical diagnostic laboratory settings without the need for multiple cytology specimens from each patient or expensive instrumentation.

The following is a detailed description of the instrument itself, as well as the results of validation tests performed to date.

\section{Materials and methods}

System overview. The majority of common staining protocols involve the application of universal reagents that are independent of the biomarker being tested and thus do not need to be applied to biopsies on an individual basis. Examples of these universal steps include deparaffinization, buffer washes, and counterstaining. These parts of the procedure can be performed in a conventional manner by applying the reagent to the entire slide. Biomarker-dependent reagents that must be applied to biopsies on an individual basis are typically much more expensive than the universal reagents, and make up 50-75\% of the overall reagent cost. As there are currently a number of systems for automating application of reagents to an entire slide (8-10), the focus of TMAS' development was the automation of the biomarker-dependent steps, specifically the application of primary and secondary antibodies and DNA probes. These steps have been automated 


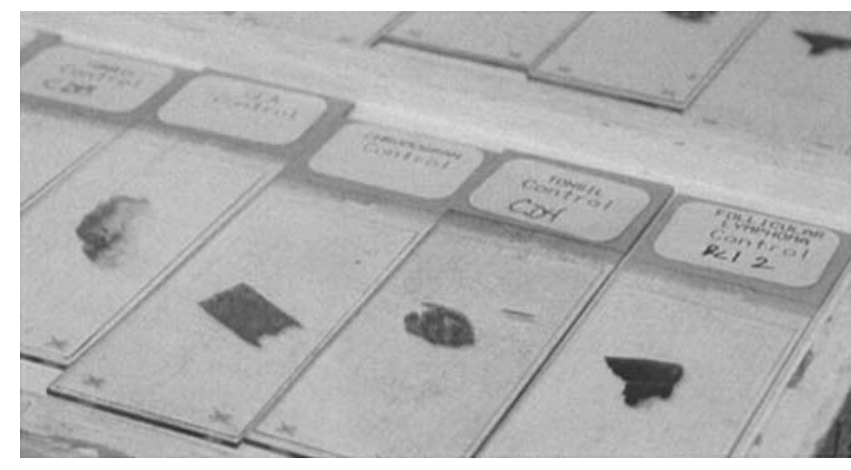

Figure 1. Single tissue section biopsy slides after undergoing a standard immunohistochemistry (IHC) staining protocol.

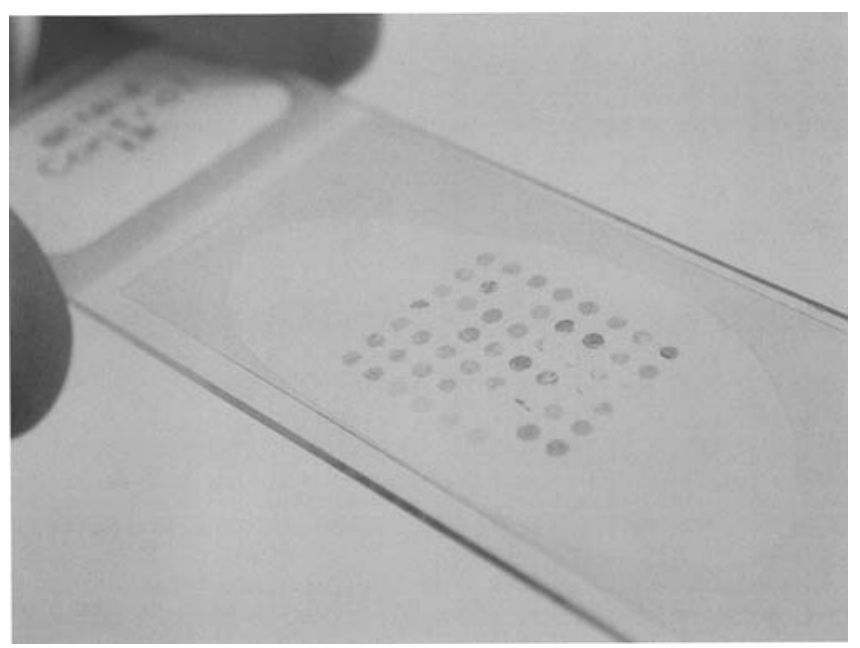

Figure 2. Tissue microarry after IHC staining using standard methods.

with TMAS while the universal steps of the protocols are carried out with conventional methods.

The core component of the TMAS system is the reagent applicator pin that was designed specifically to achieve noncontact delivery of precise volumes of reagent to individual biopsies in a TMA. The applicator pin is mounted to a triaxis linear stage (Parker Hannifin Corporation, Cleveland, USA; LX80L and MX80L) that moves the applicator pin through the staining cycle of reagent pickup, delivery, and pin cleaning (Fig. 3).

The TMA slide is held in place in a temperature-controlled slide tray that is illuminated by a bank of LEDs. A CCD camera is mounted above the slide tray for imaging and registration of the TMA biopsies to the linear stage. A combined reagent tray/cleaning station assembly holds the reagents to be applied to the TMA and contains the necessary washing and drying apparatus for cleaning the applicator pin. A data acquisition (DAQ) card connects the TMAS electronics to a PC running National Instruments LabVIEW 7.1 , and the instrument is controlled by custom LabVIEW software. Each of the components is described in detail below.

Applicator pin. The applicator pin consists of two stainless steel electrodes isolated from each other in a hydrophobic

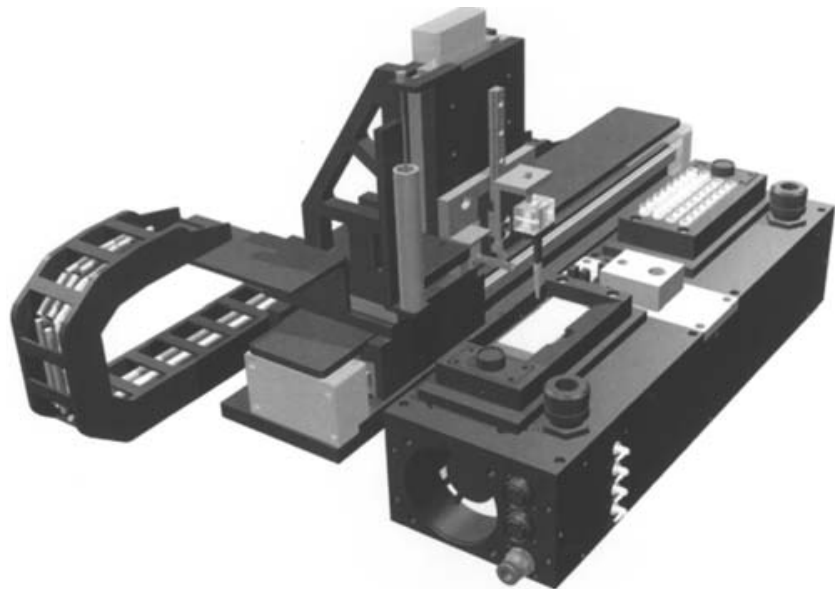

Figure 3. TMAS' linear motors, reagent tray, cleaning station, and slide tray.

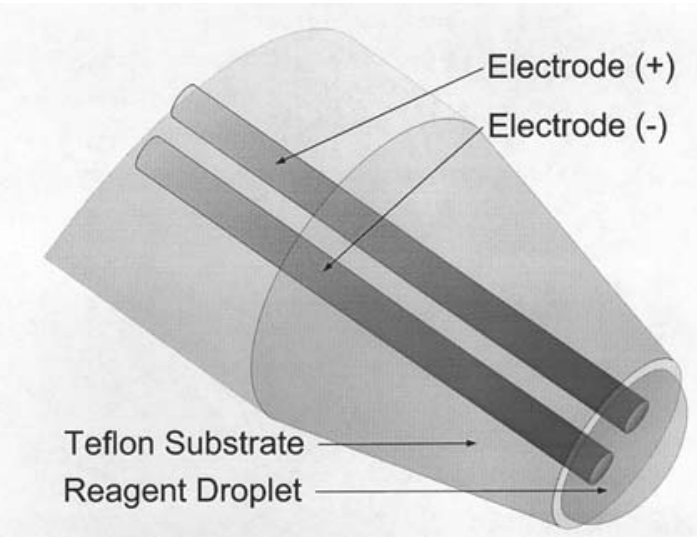

Figure 4. Detail of applicator pin tip.

Teflon ${ }^{\circledR}$ housing, as shown in Fig. 4. When the pin is brought into contact with a reagent, the hydrophobicity of the Teflon prevents the reagent from wicking up the sides of the pin, and the reagent instead forms a droplet covering the tip of the pin and bridging the electrodes. The presence of reagent on the pin tip can be determined by measuring the conductivity between the electrodes in response to an applied potential. The applicator pin is mounted to the $\mathrm{Z}$-axis (vertical axis) stage in a flexible, custom-built pin holder (Fig. 5).

A built-in limit switch allows the pin to move independently of the Z-stage and signals a collision in case of contact with the TMA slide during reagent delivery, avoiding damage to the applicator and slide.

Reagent tray and cleaning station. The reagent tray is a temperature controlled aluminum block with an array of holes machined to accommodate microcentrifuge tubes containing the specified reagents. The cleaning station consists of a small washing chamber into which the pin is lowered. The pin is first sprayed with isopropanol, followed by deionized water. Silicon tubing connects the bottom of the washing chamber to a vacuum-pumped glass bottle waste chamber. Alcohol fumes are sucked into the waste chamber, and a filter on the pump exhaust prevents them from entering the laboratory environment. The pin is then dried with a short 

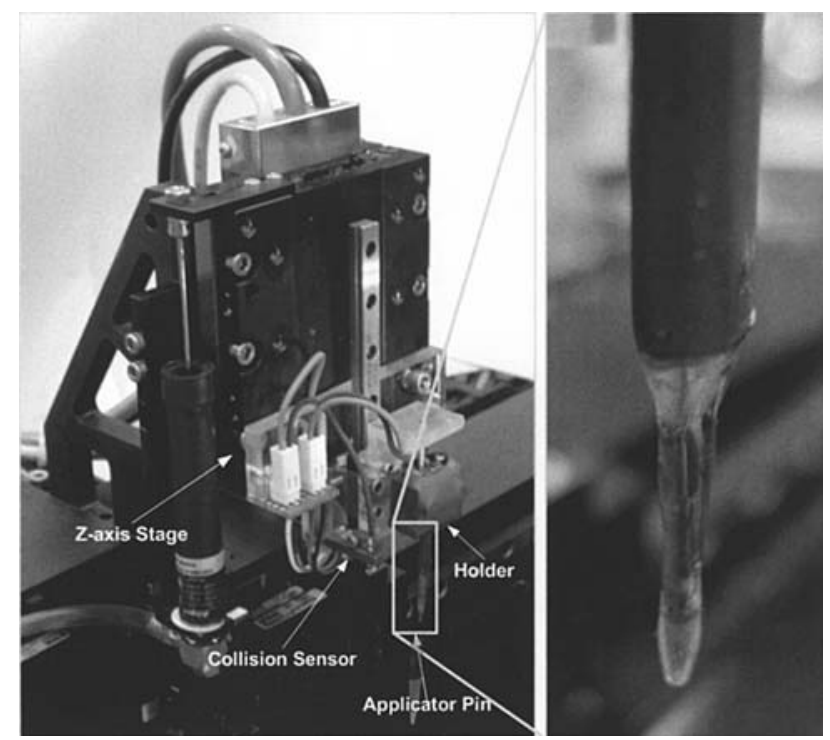

Figure 5. The applicator pin is inserted into a custom pin holder that is mounted to the vertical stage.

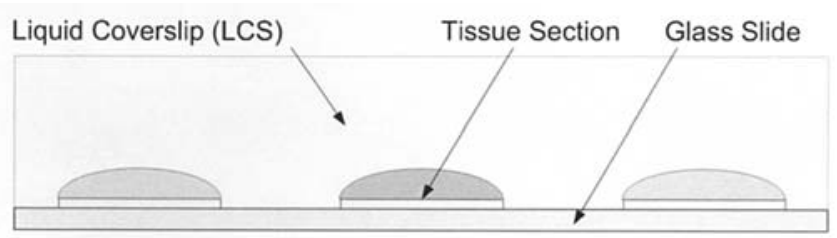

Figure 6. Three tissue sections in a TMA are shown submerged in Liquid Coverslip. The Liquid Coverslip prevents reagent (shown as bubbles above the tissue sections) from evaporating or diffusing to adjacent locations.

blast of house compressed air that is regulated to 2.5 psi and then heated. The applicator pin is cleaned between each delivery, and the entire cleaning procedure is completed in approximately $5 \mathrm{sec}$.

Slide tray. The slide tray is a custom-built mount for keeping the TMA slide held rigidly in place during imaging, registration, and reagent delivery. The slide tray contains two peltier elements to drive the temperature between $4^{\circ} \mathrm{C}$ and $95^{\circ} \mathrm{C}$ with control stability of $\pm 0.3^{\circ} \mathrm{C}$. The TMA slide rests on top of a small mirror (described in the imaging section) and is covered with approximately $10 \mathrm{ml}$ of Liquid Coverslip ${ }^{\mathrm{TM}}$ (Ventana Medical Systems, Tucson, USA; 650-010), as shown in Fig. 6. The Liquid Coverslip prevents the biopsies from dehydrating during reagent delivery and incubation, and also acts as a barrier to prevent reagents diffusing to adjacent array locations.

Lighting and imaging. In order to achieve precise delivery of reagent to the TMA biopsies it is necessary to identify and register the biopsy locations to the coordinate frame of the tri-axis stage and applicator pin. Each TMA must be addressed on an individual basis as the array size, location, and orientation with respect to the glass slide is variable. A 1/2' CCD camera (JAI Pulnix, San Jose, USA; TM-250) is mounted above the slide tray and the TMA slide is illuminated by a bank of LEDs as shown in Fig. 7. Light incident at $45^{\circ}$

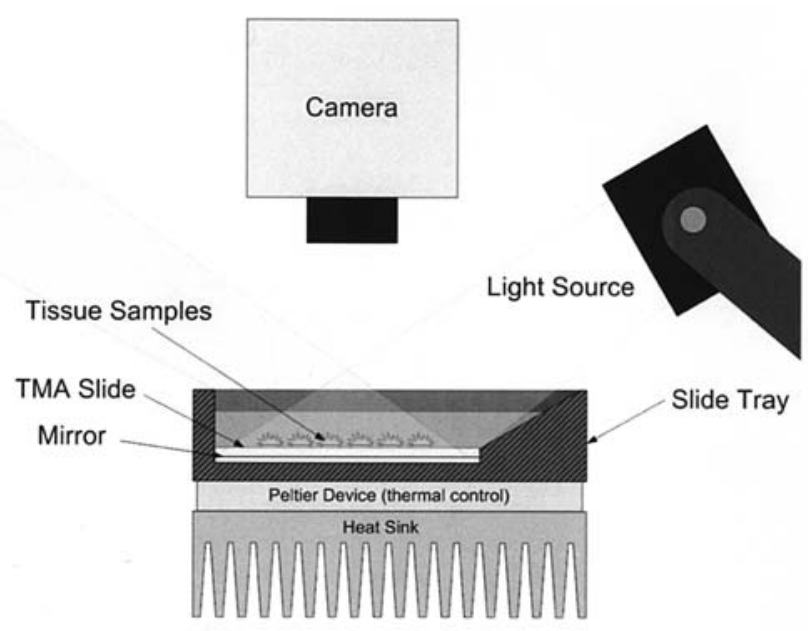

Figure 7. Cross-section of TMAS' slide tray, showing the lighting and camera arrangement.

is scattered by the biopsies in the TMA and collected by the overhead camera. The remaining light is reflected off the mirror and the black anodized finish of the slide tray provides a high contrast background to the illuminated biopsies.

Biopsy detection and registration. The image processing algorithm for automated location of the biopsies on the microscope slide was written in LabVIEW 7.1 (National Instruments Corporation, Austin, TX) using the IMAQ image processing functions. A simplified description of the detection algorithm is outlined below.

Certain user-defined threshold values are used to increase the contrast of the acquired image. A Danielsson distance map (11) is then created and used to determine the location and size of each biopsy in the TMA. Only biopsies that are circular in shape and of a predefined minimum size are considered. In this way, particulates or scratches on the glass surface are not interpreted as tissue sections in the array. The set of detected tissue biopsies can be edited manually, as described in the section on the user interface.

Registration of the biopsies is performed manually via the user interface. The black anodized finish of the slide tray has a number of laser-etched crosshairs that appear in the image of the TMA. During the start up procedure, the user is asked to click on the center of the crosshairs in the image acquired by the overhead CCD camera. The transform between the camera coordinate frame and the slide tray is then automatically determined by the control software, and this transform is used to translate the location of the biopsies from the camera frame to the slide tray frame. The slide tray coordinate frame is registered to the tri-axis stage frame mechanically, and does not change from run to run.

User interface. The user is shown an image of the TMA slide with overlaying colored circles indicating the locations of the biopsies that were identified by the biopsy detection algorithm. This display updates in real-time as the threshold values for biopsy detection are adjusted. Once the user is satisfied with the detection results, the position and size of each array location are saved to file. The user is able to add 
missed biopsies by using the mouse to 'point-and-click' on the missed locations.

The user then defines which antibodies are applied to which array locations, either one at a time, or by defining groups of biopsies. Once this procedure is complete, the reagent application process can begin. If the user wants to make changes to the reagent-biopsy allocation, the application process can be interrupted and then resumed once the changes have been made. Standard protocols can also be defined in order to reduce user input.

The interface provides access to all high-level functions of the instrument, allowing the user to interrupt and resume reagent delivery, change reagent specifications, and update temperature settings. Additionally, the interface alerts the user of any errors during the procedure such as physical contact between the applicator and the tissue samples, or incomplete reagent delivery. The interface also allows the user to predefine actions in case of errors or to deal with each error case individually. It also provides the user with realtime information such as whether reagent is present on the applicator, which tissue sample is being targeted, which reagent is being used and the current position of the applicator pin.

Central control. A PC (Pentium 4, 512 MB RAM) running Windows XP and LabVIEW 7.1 acts as the central control unit for TMAS.

Control electronics. The central component of the control electronics is a National Instruments data acquisition (DAQ) card (National Instruments Corporation; PCI-6036-E) and an associated breakout box (CB-69LP).

Staining procedure. The following is a description of a typical IHC staining protocol (formalin fixed, paraffin-embedded tissue sections) that has been modified for use with the TMAS instrument. Specific attention has been given to the steps that have been altered to allow for automation using TMAS - these steps are indicated as (TMAS adapted). This modified protocol was derived from the manual protocol used at the British Columbia Cancer Agency (BCCA) IHC Lab (12).

\section{Preparation}

a) Deparaffinization in xylene

b) Clear xylene in ethanol

2. Antigen retrieval

3. Block endogenous peroxide activity

4. Buffer wash (TMAS adapted)

Typically, excess buffer is simply wiped from the edges of the slide, leaving a thin layer of buffer covering the entire microarray to keep the tissue sections hydrated until primary antibody is applied in step 6 . When staining with TMAS, however, residual buffer that bridges adjacent array elements would allow reagent cross-talk between the array elements, leading to contamination between assays on individual elements. In order to ensure that each array location is properly hydrated, and yet individually isolated, a squirt bottle is used to rinse the slide while it is held at an angle, and the buffer is allowed to run off. The slide is then manually inspected to ensure that there are no bridging droplets left on the slide.

\section{Liquid Coverslip (TMAS adapted)}

The amount of moisture maintained in each tissue sample after the modified buffer wash (step 4) is roughly $50 \mathrm{nl}$ and evaporates at room temperature in $<1 \mathrm{~min}$. In accordance with established protocols, precaution must be taken to ensure that the tissue samples are not permitted to dry out at any point during the staining procedure. Conventionally, this is accomplished by covering the entire array with enough reagent to prevent dehydration during the incubation; however, this allows contamination between tissue samples, and is not an acceptable technique for the TMAS system. Instead, the entire slide is covered in a commercially available Liquid Coverslip, which is designed for use in Ventana autostainers for high temperature incubations. Because the Liquid Coverslip is oil based, it does not interact with the aqueous reagents, but instead forms a vapour barrier preventing evaporation, even at temperatures nearing $100^{\circ} \mathrm{C}$. In addition to keeping the tissue samples from dehydrating, the Liquid Coverslip also acts to eliminate reagent cross-talk between the tissue samples as it keeps each array location isolated and surrounded by a barrier of oil.

6. Primary antibodies (TMAS adapted)

a) The various primary antibodies are applied in $20 \mathrm{nl}$ aliquots to the individual array elements. It takes roughly $12 \mathrm{sec}$ (including cleaning) to deliver one droplet to a single biopsy in the array. Consequently, depending on the number of samples in the array, there could be a significant time lag between the first and last delivery cycles. The slide is held at $4^{\circ} \mathrm{C}$ during delivery so that the effective incubation time is the same across all biopsies.

b) The slide tray is then raised to room temperature for the duration of the incubation. Optimal incubation times can differ between antibodies and are dependent on antibody concentration. Generally all assays performed on the same slide must have the same incubation time; therefore, the antibody concentration can be calibrated to allow for optimal staining when using TMAS. Alternatively, primary antibodies can be applied to specific array locations after the incubation period has started to allow for shorter incubation times.

\section{7. (Steps 4 and 5 are repeated)}

8. Secondary antibodies (TMAS adapted)

a) The slide is returned to $4^{\circ} \mathrm{C}$ while the appropriate secondary antibodies/labeled polymers are applied to each array location.

b) The slide is then returned to room temperature for the duration of the incubation (usually $\sim 30 \mathrm{~min}$ ). As described in step 6 , concentration of secondary antibodies can be varied to allow for uniform effective incubation time.

9. Chromogenic particles (DAB)

10. Haematoxylin counterstain

\section{Results}

Imaging performance. Cases in which the software is likely to overlook elements of the tissue array are when tissue sections have been deformed or are otherwise misshapen; this proves to be of little consequence as deformed tissue samples are usually not useful for histological analysis. Provided that the samples have undergone the proper washing procedure and have been adequately hydrated, $<1$ in 100 analytically useful tissue samples are overlooked by the system. In 
A

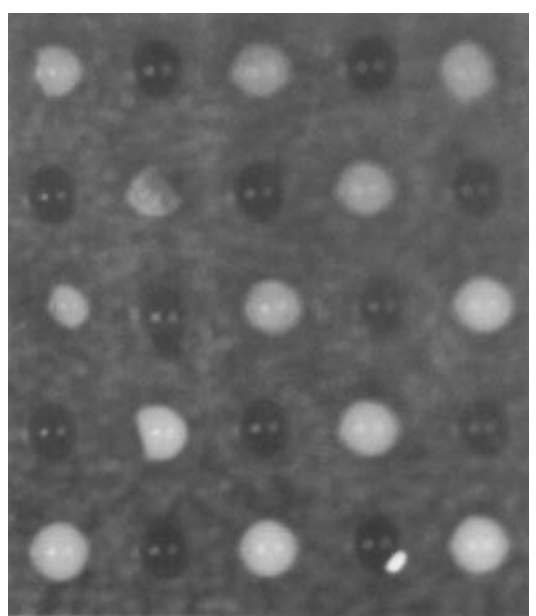

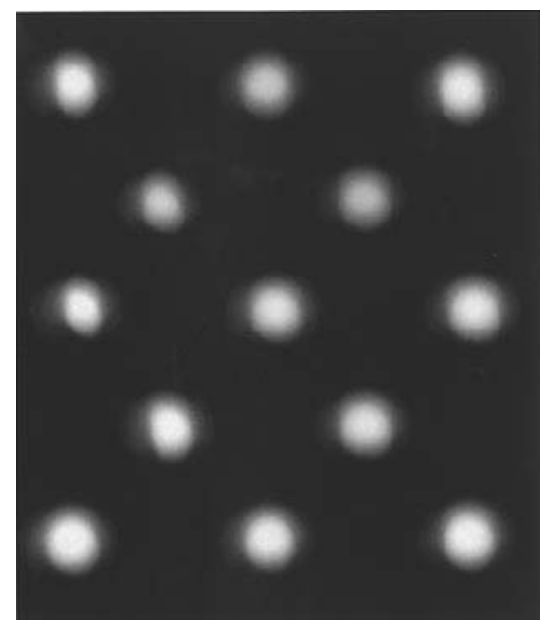

Figure 8. (A) Photograph of a 5x5 TMA section spotted with fluorescein and blue dye in a checkerboard pattern. (B) Fluorescence image of the same section.
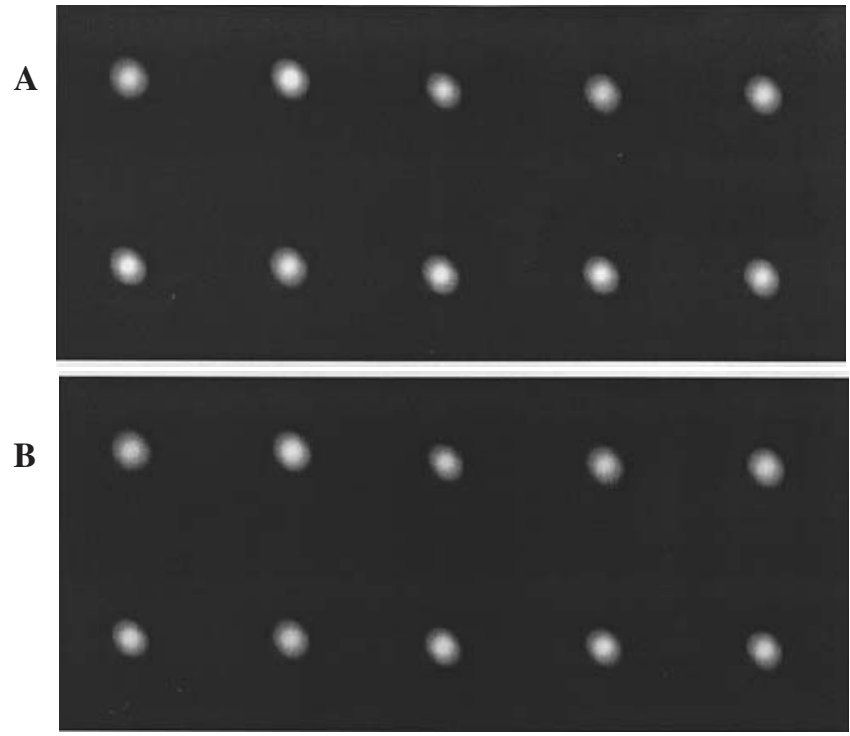

Figure 9. A 5x2 TMA was spotted with fluorescein dye and imaged at $10 \mathrm{~min}$ intervals over a 90 min period. The image in (A) was taken immediately after the fluorescein was spotted. (B) Shows an image taken 90 min later.

such cases however, the user is able to manually add those missed locations using the point-and-click method described previously. False biopsy identifications are completely avoided by ensuring that the slide tray is kept clean and free of particulate impurities that may scatter light to the CCD camera.

Reagent application and isolation. Tests were conducted to examine two different forms of possible contamination between adjacent biopsies: inadequate cleaning of the applicator pin between reagent deliveries and diffusion of reagent through the Liquid Coverslip.

To test the adequacy of the cleaning procedure the $5 \times 5$ array shown in Fig. 8 was spotted with a $0.5 \mathrm{mg} / \mathrm{ml}$ fluorescein solution and blue dye as a control. Deliveries were done from left-to-right, top-to-bottom, alternating between the two solutions. The applicator was cleaned after each delivery so that inadequate cleaning would be indicated by fluorescence from the tissue samples that were spotted only

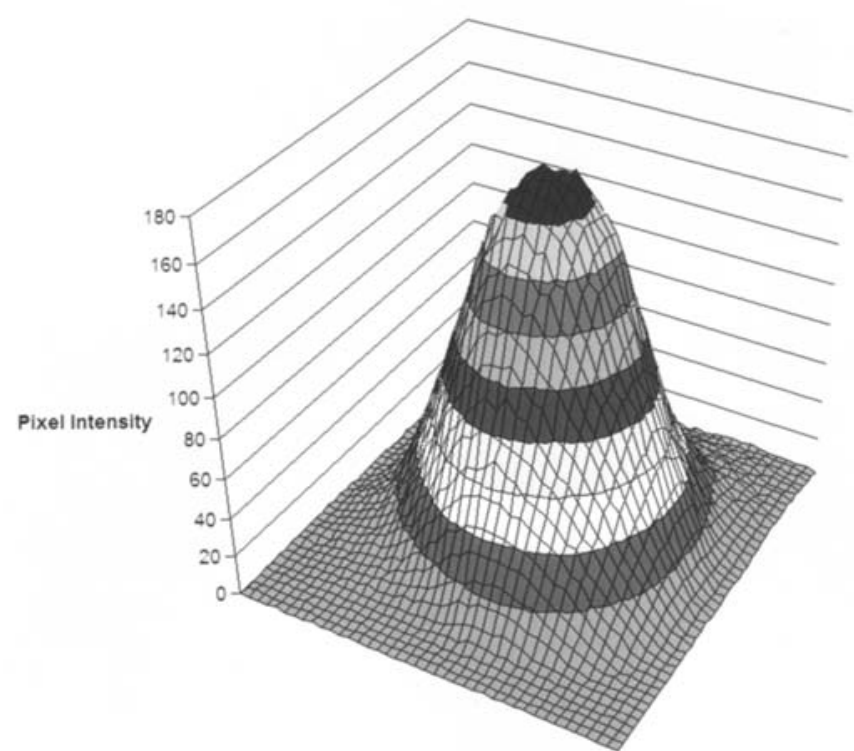

Figure 10. A surface plot of the pixel intensity of the top left spot in Fig. 9A immediately after the fluorescein was spotted. The pixel intensity ranges from 0 (black) to 255 (white).

with blue dye. All fluorescence images of fluorescein-spotted arrays were taken using UV excitation at $365 \mathrm{~nm}$ and a $\pm 67 \mathrm{~nm}$ band pass emission filter centered at $555 \mathrm{~nm}$.

Pixel intensity levels were recorded at the locations that were spotted with blue dye and compared with background fluorescent levels. The results showed no measurable residual fluorescence in those array locations, indicating that the pin cleaning procedure was adequate to prevent contamination.

Fig. 9 shows a $5 \times 2$ array which was spotted with $0.5 \mathrm{mg} /$ $\mathrm{ml}$ fluorescein. After spotting the slide was imaged at 10-min intervals over a period of $90 \mathrm{~min}$. Diffusion of the fluorescein solution into the Liquid Coverslip was measured by monitoring the intensity profile of each array location throughout the 90-min period. Background fluorescent levels were calculated and subtracted from the images, and surface plots of pixel intensity, as shown in Fig. 10, were constructed for 


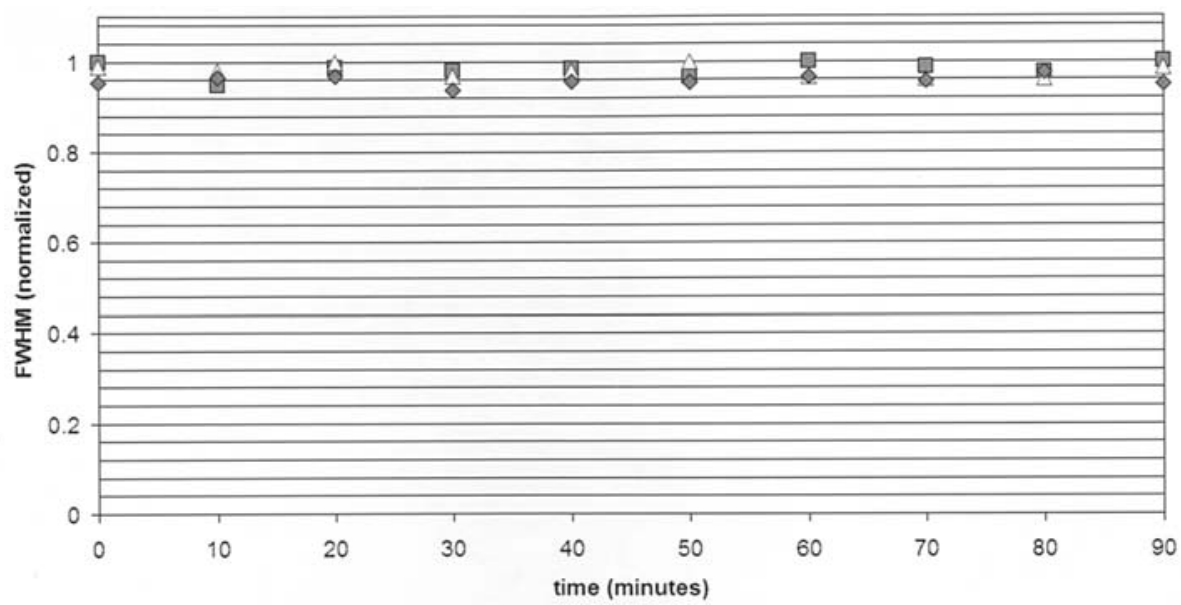

Figure 11. Normalized FWHM variation in 3 of the 10 spots shown in Fig. 9 over time. No correlation is found between FWHM and time after spotting, indicating no measureable diffusion during the 90 -min incubation.

A
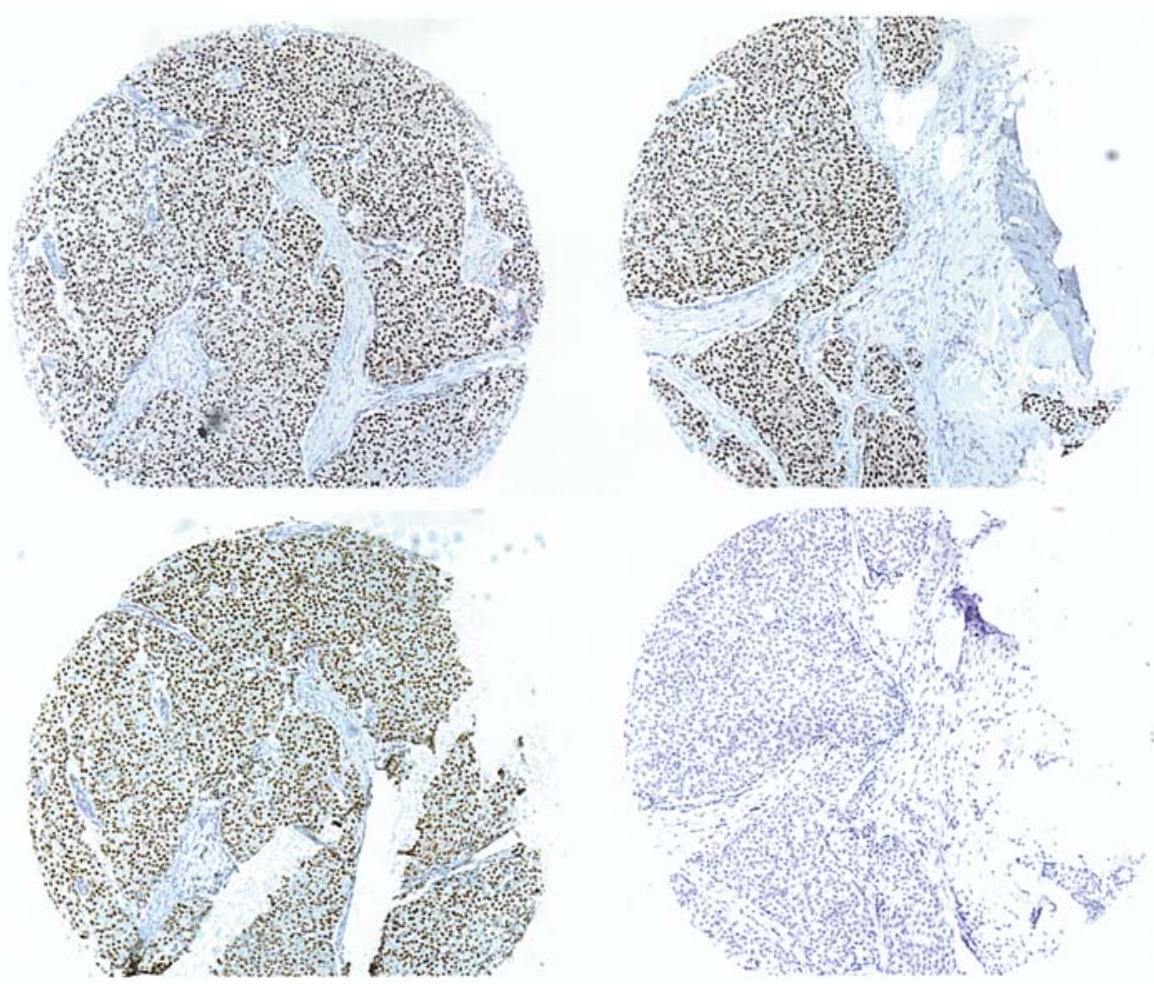

Figure 12. (A) Adjacent tissue sections from a TMA slide were stained for the estrogen receptor alpha protein following a manual protocol (3). (B) Sections of the same two adjacent tissue cores as shown in (A) also stained for the estrogen-receptor, this time using TMAS. The left-hand section was stained using the TMAS-adjusted protocol outlined above and shows good positive staining as expected from the manual results. The right-hand section was purposely not spotted with primary antibody in order to test for reagent cross-talk between adjacent tissue sections. The lack of positive staining indicates successful isolation of each core. These images have been cropped to reduce empty slide space between biopsy sections.

each spot in each of the 10 pictures taken over the 90 -min period.

The diffusion of the fluorescein solution was measured by monitoring the full-width at half-maximum (FWHM) intensity of these surface plots as time progressed throughout the incubation. Fig. 11 shows the normalized FWHM values for 3 randomly selected spots in the array over the course of the 90-min incubation. No correlation is found between the FWHM and time after spotting, indicating no measurable diffusion of the fluorescein solution into the Liquid Coverslip.
These results are supported by other tests (data not shown), allowing us to conclude that any diffusion of aqueous reagents into the Liquid Coverslip is of no practical concern for TMAS.

Immunohistochemical results. IHC testing of the TMAS prototype to date has consisted of applying primary and secondary antibodies. FISH staining (VYSIS, PathVysion HER2 DNA Probe Kit) has also been performed successfully using TMAS to apply the DNA probe individually to each tissue sample in the array. In both cases staining has been 
primarily successful but indicates that further protocol optimization is required to obtain consistent staining required for clinical use. Working alongside technologists at the BCCA and other collaborator sites, this protocol optimization is currently the primary objective of the project.

Fig. 12 shows two sets of images of adjacent TMA biopsies that were IHC stained for the estrogen receptor alpha protein. The slide from which the image in Fig. 12A was taken was stained following a well-established manual protocol (3). Both sections are graded as highly positive (3+), which is evident from the heavy positive (brown) staining. Fig. 12B shows sections of the same two adjacent biopsy cores that were stained for estrogen receptor alpha protein using TMAS. The left-hand section in Fig. 12B was stained following a TMAS-adjusted protocol as outlined above. This section shows good positive staining in accord with the results of manual staining in Fig. 12A. The right-hand section was stained following the same TMAS-adjusted protocol, with the exception that a buffer solution was purposely applied instead of primary antibody. With no primary antibody, any positive staining in this section would have indicated falsepositive staining or undesired reagent cross-talk. The absence of any such staining in this section is indicative of TMAS' ability to isolate individual sections in a TMA for multiplexing assays.

Applicator pin lifetime. Exact service lifetime of each applicator depends on workload, and also varies from pin to pin to due to the manual nature of the fabrication process. Average workload during two years of prototyping has been roughly one slide per day, with each slide consisting of roughly 100 tissue sections. Under these conditions, service lifetime is typically 8 weeks, ranging from as few as 2 weeks, to as much as 4 months. While the exact mechanisms that lead to pin degradation are not fully understood, an extensive study to investigate factors that effect service lifetime will take place in the fall of 2006. The results of this study will be used to establish a more rigorous pin design and a more uniform fabrication process that will ensure consistent and parameterized pin performance.

\section{Discussion}

The TMAS instrument has been shown capable of exploiting TMA platforms in order to provide higher assay throughput, with more flexibility and significantly lower cost per assay. Specifically, TMAS allows each array location in a TMA to be individually addressed-permitting hundreds of histological assays to be performed in parallel on a single microscope slide.

The volumes of reagent used per assay with TMAS are particularly noteworthy. Conventional protocols (whether done manually or with an automated staining machine) use approximately $200 \mu 1$ of reagent per slide. On a TMA with 200 tissue sections, this amounts to $1 \mu 1$ per individual biopsy, and requires all biopsies to undergo the same assay. In contrast, the volume of reagent that is transferred during each TMAS delivery is approximately $20 \mathrm{nl}$. If more reagent is required for certain tissue samples, this can be accomplished by repeat deliveries. Assuming that 5 reagent deliveries are made to each tissue sample (in testing, a typical biopsy required 2 deliveries), then $100 \mathrm{nl}$ of reagent is used per tissue sample - approximately 10 times less reagent per assay than conventional protocols. Because the amount of reagent used per slide scales with the number of tissue samples in the array, cost savings are even more significant for arrays with fewer elements, since conventional methods use a relatively fixed volume irrespective of the density of the array.

As reagent costs for IHC-based assays are on the order of tens of dollars per slide, it is easy to see how significant reduction in reagent consumption would lead to considerable overall savings in a high throughput environment. By way of example, the Histology Lab at the British Columbia Cancer Agency in Vancouver, British Columbia stains over 30,000 slides every year with a reagent budget of approximately 500,000 Canadian dollars.

Ultimately, extending this instrument for use with cytology arrays derived from a single patient's fine needle aspiration biopsy introduces the potential for extremely rapid (overnight) molecular profiling of a patient to help guide treatment decisions.

\section{Acknowledgements}

The authors wish to thank the following people from the BC Cancer Agency: Yin Ping Lee and Chris Dawe, Melinda Miller for providing technical assistance and invaluable guidance, and Bev Thomas and Dr David Huntsman for coordinating these efforts and providing supplies and reagents. We also acknowledge Miti Isbasescu, Joel Pel, Nahid Jetha, and Mathew Wiggin of the UBC Applied Biophysics Laboratory and the Genome BC Technology Development Platform for assistance and useful discussions. This work is funded by Genome British Columbia.

\section{References}

1. Kononen J, Bubendorf L, Kallioniemi A, et al: Tissue microarrays for high-throughput molecular profiling of tumor specimens. Nat Med $4: 844-847,1998$.

2. Hsu FD, Nielsen TO, Alkushi A, et al: Tissue microarrays are an effective quality assurance tool for diagnostic immunohistochemistry. Mod Pathol 15: 1374-1380, 2002.

3. Henshall S: Tissue microarrays. J Mammary Gland Biol Neoplasia 8: 347-358, 2003.

4. Chen W and Foran DJ: Advances in cancer tissue microarray technology: towards improved understanding and diagnostics. Anal Chim Acta 564: 74-81, 2006

5. Torhorst J, Bucher C, Kononen J, et al: Tissue microarrays for rapid linking of molecular changes to clinical endpoints. Am J Pathol 159: 2249-2256, 2001.

6. Schraml P, Kononen J, Bubendorf L, et al: Tissue microarrays for gene amplification surveys in many different tumor types. Clin Cancer Res 5: 1966-1975, 1999.

7. Zhang DH, Salto-Tellez M, Putti TC, Do E and Koay ESC: Reliability of tissue microarrays in detecting protein expression and gene amplification in breast cancer. Mod Pathol 16: 79-84, 2003.

8. Ventana Medical Systems Inc: Ventana Instrument General Overview (on-line). http://www.ventanamed.com/products/ instruments/instruments.html, 2006.

9. Dako Denmark A/S: Dako Autostainer Universal Staining System (on-line). http://www.dako.ca/index/prod_search/prod_ groups.htm?productareaid=18, 2006.

10. BioGenex Inc: Autmation Systems (on-line). http://www. biogenex.com/products/automation.asp, 2006.

11. Danielsson PE: Euclidean distance mapping. Computer Graphics and Image Processing 14: 227-248, 1980.

12. British Columbia Cancer Agency, Department of Pathology and Medicine. Form \# HIJA 84oc. 